\title{
Implementación de un modelo teórico a Priori de competencia matemática asociado al aprendizaje de un objeto matemático
}

\section{Implementation of an a Priori theoretical model of mathematical competence associated with the learning of a mathematical object}

Bernardo García-Quiroga'
Arnulfo Coronado $^{2}$
Albeiro Giraldo-Ospina $^{3}$

Recibido: octubre 13 de 2016 Aceptado: diciembre 27 de 2016

\section{Resumen}

Este artículo es producto de una investigación cuyo objetivo fue el de apoyar al docente de matemáticas con orientaciones didácticas para promover el desarrollo de las competencias matemáticas de los estudiantes. En el trabajo investigativo se utilizó la complementariedad metodológica y se asumieron orientaciones específicas de la investigación acción participación. El modelo teórico a priori de competencia matemática se formuló con los aportes de los investigadores Bishop, Sfard, D’Amore, Godino, Fandiño y Solar. Este modelo se aplicó durante el primer semestre de 2015 y se focalizó en una secuencia de tareas matemáticas, centrada en el actual proceso de paz entre las Fuerzas Armadas Revolucionarias de Colombia, FARC, y el gobierno colombiano. La implementación del modelo aludido se hizo con la colaboración de estudiantes de grado sexto de la Institución Educativa Jorge Eliécer Gaitán de Florencia, Caquetá. El modelo teórico a priori formulado trascendió el aspecto cognitivo; su aplicación contribuyó al desarrollo de procesos matemáticos, afectivos y de tendencia de acción para compartir y desarrollar el significado matemático en clase. De esta manera, se constituyó en una adecuada mediación didáctica que orienta al profesor en su propósito de desarrollar las Competencias Matemáticas de los estudiantes.

Palabras clave: competencia matemática, modelo de competencia matemática, objeto matemático, significado matemático.

\begin{abstract}
This article is the product of a research addressed to support the mathematics teacher with didactic orientations to promote the development of the mathematical competences of the students. In the research work a methodological complementarity was used and specific guidelines of Participatory Action Research were taken on. The a priori theoretical model of mathematical competence was formulated with the contributions of researchers like Bishop, Sfard, D'Amore, Godino, Fandiño and Solar. This model was applied during the first half of 2015 and focused on a sequence of mathematical tasks centred on the current peace process between the Revolutionary Armed Forces of Colombia, FARC, and the Colombian government. The implementation of the model was made with the collaboration of sixth grade students of the Educational Institution Jorge Eliécer Gaitán of Florencia, Caquetá. The a priori theoretical model formulated transcended the cognitive aspect; its appli-
\end{abstract}

1 Licenciado en Matemáticas y Física, Doctor en Ciencias Pedagógicas, Universidad de la Amazonía, Colombia. E-mail: bgarciaquiroga@ hotmail.com

2 Licenciado en Matemáticas y Física, Magíster en Docencia de la Matemática, Universidad de la Amazonía, Colombia. E-mail: arcoronado_123@yahoo.es

3 Licenciado en Matemáticas y Física, Magíster en Docencia de la Matemática, Universidad de la Amazonía, Colombia. E-mail: albeiro70@gmail.com 
cation contributed to the development of mathematical processes, affective and action trend processes for sharing and developing mathematical meaning in class. In this way, it was constituted in an adequate didactic mediation that guides the teacher in his / her purpose of developing the mathematical competences of the students.

Keywords: mathematical competence, mathematical competence model, mathematical object, mathematical meaning.

\section{Introducción}

El desarrollo de competencias y en particular de Competencias Matemáticas, CM, hace parte del discurso curricular oficial colombiano desde 2006 $y$, desde algunas décadas antes, en el plano internacional (Cerón-Álvarez, Mesa-Laverde, \& Rojas-Morales, 2012; Montes-Miranda, 2013). Competencia es un concepto complejo y polémico, pero básico para enfrentar los cambios y retos, especialmente en el proceso de formación humana y, en general, en el campo educativo. Hoy, las competencias se han consolidado como un importante organizador curricular que debe estudiarse a profundidad.

No obstante lo anterior, la implementación de un currículo basado en el enfoque por competencias presenta diferentes problemas. Uno de ellos radica en que el discurso sobre CM presenta desarrollos teóricos que aún no han incidido lo esperado en las prácticas de aula (Piratoba-Gil., \& Rojas-Morales, 2014; Riscanevo-Espitia, 2016). En este sentido, Solar (2009) expresa que: "entre los profesores existe una sensación de falta de herramientas para promover el desarrollo de las competencias y desde la óptica didáctica no existe una cantidad de experiencias empíricas para su estudio, menos aún, resultados de investigación".

Otro problema es la ausencia de conceptualización personal e institucional sobre Competencia; esto fue evidente en los Proyectos Educativos Institucionales, PEl, en las instituciones educativas es- tudiadas en el departamento de Caquetá, Colombia. Igualmente, fue representativa la restricción del concepto de competencia matemática por parte de los profesores, al "saber hacer en contexto" (García et al., 2012).

Los resultados de los estudiantes caqueteños en las pruebas Saber, con promedios inferiores a los nacionales según diagnóstico realizado por García y Coronado para la Universidad Abierta y a Distancia, UNAD, en el año 2015, son otro factor que, junto a los anteriores problemas, constituyen la base argumentativa de la pregunta de investigación: ¿Cómo contribuir al desarrollo de las CM, representar, razonar y argumentar y comunicar, en los estudiantes de grado sexto de la Institución Educativa Jorge Eliécer Gaitán?.

Apoyados en un modelo de competencia matemática elaborado por Solar (2009) y focalizado en la enseñanza, esta investigación propone como hipótesis de trabajo la construcción e implementación de un Modelo Teórico a Priori, MTP, de competencia matemática centrado en la actividad matemática de aprendizaje del estudiante.

El MTP construido con los aportes de Solar (2009), D’Amore, Godino y Fandiño (2008), Sfard (2008), Fandiño (2006) y Bishop (2005), entre otros, y que se expondrá en los referentes teóricos y conceptuales, se implementó con una secuencia de tareas matemáticas centrada en el actual proceso de paz entre las Fuerzas Armadas Revolucionarias de Colombia, FARC, y el gobierno colombiano, y articulada al objeto matemático tanto por ciento. Los datos sobre la actividad matemática de aprendizaje que la secuencia de tareas generó, se sistematizaron y valoraron a partir del concepto asumido de competencia matemática y, en particular, a partir de la conceptualización construida para las CM: representar, razonar y argumentar, y comunicar. Los métodos utilizados, los resultados obtenidos y la correspondiente discusión, junto con las conclusiones, se dan a conocer en las secciones subsiguientes. 


\section{Referentes teóricos y metodología}

\subsection{Sobre el Modelo Teórico a Priori de Competen- cia Matemática}

El concepto de competencia matemática asumido se constituyó en la base del MTP formulado e implementado, pues este concepto incluye los elementos sustanciales del modelo. Se comparte con Chacín (2008), que el modelo es considerado como un espacio conceptual que propicia la comprensión de la realidad compleja, ya que selecciona los elementos más representativos y establece relaciones entre ellos. Solar (2009) asume la "cultura matemática", las CM, los procesos matemáticos, las tareas matemáticas y los niveles de complejidad, como principios de su noción de competencia matemática; luego los adopta como componentes de su modelo de competencia matemática, ver Figura 1.

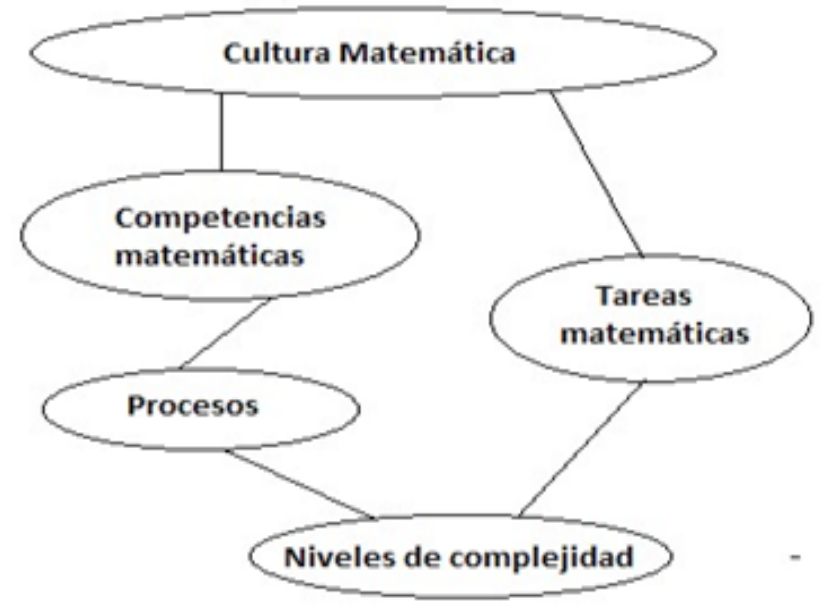

Figura 1. Modelo de competencia matemática (Solar, 2009, p. 57).

Desde los planteamientos de la Organización para la Cooperación y Desarrollo Económico, OCDE (2003), Solar (2009) considera la competencia matemática como "dominio de estudio (...), y supone un modo global de entender y hacer matemáticas, como a su vez comprender la naturaleza del cono- cimiento matemático". Precisa Solar (2009) que las CM son procesos matemáticos específicos. Desde esta perspectiva para Solar (2009), pensar y razonar, argumentar, comunicar, construcción de modelos, plantear y resolver problemas, representar, utilizar lenguaje y operaciones simbólicas formales y técnicas, y el empleo de material y herramientas de apoyo, son $\mathrm{CM}$, de modo que su dominio permite el desarrollo de la competencia matemática en general.

Solar (2009), centra el concepto de competencia matemática en el desarrollo del aspecto cognitivo del ser humano, en el conocimiento de las disciplinas científicas, en este caso en el conocimiento y uso social de las matemáticas.

De manera complementaria para García, Acevedo y Jurado (2003), el concepto de competencia en una de sus dimensiones tiene relación con la educación integral y la formación de seres humanos críticos. En este sentido, Gascón (2011) expresa que el enfoque por competencias centra su atención en la formación integral del estudiante y lo direcciona hacia un ejercicio profesional, social y cívico.

Según Fandiño (2006), se necesita una concepción de competencia matemática que supere el solo "saber hacer en contexto". Se requiere un concepto que integre de manera interrelacionada componentes presentes en toda competencia tales como el saber qué, el saber qué hacer y el saber cómo, cuándo y por qué hacerlo. En esta misma dirección, Vasco-Uribe (2013), en su conferencia "¿Disposiciones, capacidades, o competencias?", expresó que a cambio de no aceptar los discursos sobre competencias por su ambigüedad y procedencia, corresponde a la comunidad académica reelaborar un concepto potente de competencia articulado de manera significativa a los diferentes aspectos del desarrollo humano.

Para D'Amore, Godino y Fandiño (2008), las CM poseen una base cognitiva disciplinar y unos contenidos matemáticos como medio de promoción 
y desarrollo. Puntualizan que la competencia matemática como concepto complejo, dinámico y polisémico se compone de tres aspectos: el cognitivo, conocimiento de la disciplina; el afectivo, disposición, voluntad, deseo de dar respuesta a un requerimiento (interno o externo); y la tendencia de acción, persistencia, continuidad y dedicación.

De otro lado, el desarrollo de las CM del estudiante está estrechamente ligado con la calidad de su actividad matemática de aprendizaje. En este sentido, Sfard (2008), en una ruptura epistemológica y didáctica con la "metáfora de la adquisición", plantea la "metáfora de la participación" como una alternativa para resignificar la actividad matemática de aprendizaje del estudiante, especialmente, en términos de resignificar el aprendizaje como un discurso de complejidad creciente con el cual el estudiante participa. Participación entendida como un proceso de aprendizaje que integra la clase como comunidad de aprendizaje y hace propia la cultura matemática de dicha comunidad. En términos de la autora:
(...) aprender matemáticas ahora se concibe como un proceso de convertirse en miembro de una comunidad matemática. Esto implica, sobre todo, la habilidad de comunicarse en el lenguaje de esta comunidad y de actuar según sus normas particulares (...). El aprendiz pasa de ser un empresario solitario a ser parte integral de un equipo. (Sfard, 2008)

Con base en los referentes teóricos expuestos, se asumió la competencia matemática como un proceso de participación en el que los estudiantes movilizan aspectos de su desarrollo humano (cognitivos, afectivos y de tendencia de acción), con el propósito de intervenir en los diferentes entornos en los que realizan sus proyectos de vida y se requiera de procesos matemáticos y no matemáticos. Concepto de competencia matemática que, en concordancia con lo expresado al inicio del presente apartado, "iluminó" la elaboración de un MTP que se explica a continuación y se ilustra en la Figura 2, junto con la Tabla 1 que corresponde a la tabla de valoración.

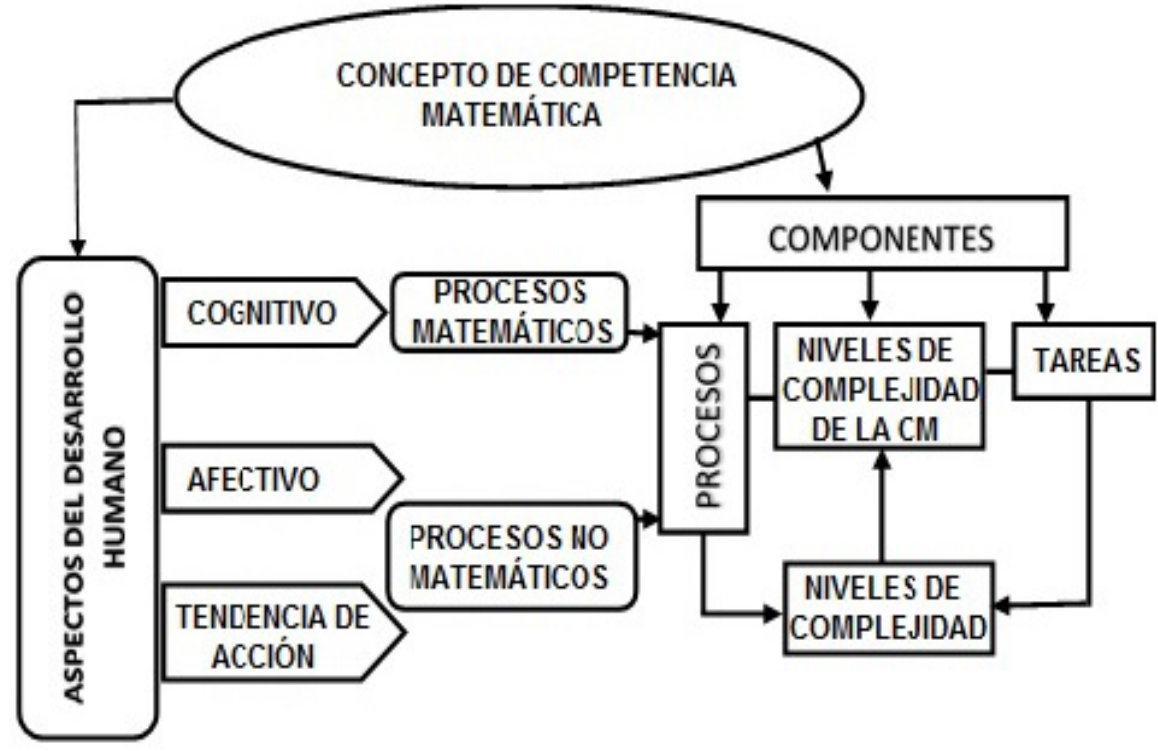

Figura 2. Modelo teórico a priori de competencia matemática.

Entonces, "Este modelo se asumió como una estructura para organizar, describir, explicar y articu- lar los componentes de la competencia matemática con la actividad matemática de aprendizaje, los 
objetivos de las tareas matemáticas y las formas de evaluación" (Solar et al., 2014). Su finalidad, contribuir a planificar el desarrollo coherente y progresivo del proceso de movilización de las competencias matemáticas cuando el estudiante resuelve tareas y desarrolla procesos cognitivos, afectivos y de tendencia de acción con complejidad creciente. Su carácter de "a priori" se explica en el modelo porque sus diferentes componentes fueron concebidos y asumidos de manera teórica y previa al proceso de implementación en el trabajo de aula.

El MTP adoptado es el artificio para la articulación de las tareas matemáticas, los procesos matemáticos y los niveles de complejidad con la actividad matemática de aprendizaje del estudiante. Ello significa que el proceso de desarrollo de las CM del estudiante se describe, explica y caracteriza, con apoyo del modelo, a partir de la calidad de las tareas matemáticas que se proponen al estudiante. Los procesos matemáticos y no matemáticos asociados a cada una de las CM, y los niveles de complejidad, junto con la actividad matemática de aprendizaje del estudiante, posibilitan la valoración y caracterización del proceso de desarrollo de las CM del estudiante (García et al., 2013).

De Solar (2009) se adoptan, como componentes del MTP de competencia matemática, los procesos matemáticos, las tareas matemáticas y los niveles de complejidad. Se comparte con Abrantes (2001, citado por Solar, 2011), que un proceso matemático expresa la manera de actuar de un estudiante cuando hace matemáticas.

Lo anterior, en concordancia con lo expresado por D’Amore, Godino y Fandiño (2008), se reconoce que en ese actuar, en ese participar, además del aspecto cognitivo (conocimiento de la disciplina matemática) y de procesos específicos matemáticos, existen otros aspectos del desarrollo humano que intervienen en el desarrollo de las CM como son el aspecto afectivo y de tendencia acción, a los cuales, se encuentran asociados otros procesos no matemáticos como son la disposición y la persistencia entre otros. Sin estos procesos, un es- tudiante difícilmente desarrollará procesos matemáticos con gusto, voluntad e inclinación cultural favorable a participar (García, Coronado, \& Giraldo, 2015). Como lo manifiestan D’Amore, Godino y Fandiño (2008): “¿Qué sería de una competencia sin el deseo, sin la voluntad y sin el gusto de hacer uso de ella?".

De esta manera, el MTP propuesto y aplicado, va más allá del aspecto cognitivo de las $\mathrm{CM}$; reconoce y legitima aspectos afectivos, de tendencia de acción, de uso social de las matemáticas como factores humanos a desarrollar. No basta con conocer las matemáticas, saberlas utilizar o resolver problemas en ellas y con ellas para afirmar que se es matemáticamente competente. Nuestra concepción asumida de $\mathrm{CM}$, implica que también es muy importante que el estudiante sea competente con las matemáticas como ciudadano, en su entorno sociocultural inmediato.

La presente investigación, sin desconocer la existencia de otras CM y las articulaciones que existen entre ellas, se focalizó en las CM razonar y argumentar, representar y comunicar. Las consultas realizadas sobre estas (Kaput, 1987; Duval, 1998; Goldin, \& Shteingold, 2001; Niss, \& Højgaard, 2011; Duval, 1999; NCTM, 2000; Espinoza et. Al., 2009; OCDE, 2006; Rico, 1995; Stacey, 1989; Balachef, 2000; Sierpinska, 1995), indican que a ellas, en el aspecto cognitivo, se asocian procesos matemáticos como describir e interpretar, aplicar, comunicar, argumentar, proponer, codificar, descodificar y traducir; los cuales, junto con procesos no matemáticos como disposición y persistencia, asociados al aspecto afectivo y de tendencia de acción, respectivamente, hacen parte del componente procesos del MTP elaborado, ver Tabla 1.

En concordancia con lo expuesto, en la base de cada competencia están los procesos (matemáticos y no matemáticos), los cuales, en un enfoque por competencias se constituyen en organizadores del currículo. Los contenidos matemáticos como elementos del dominio matemático se deben "poner al servicio" del desarrollo de los aspec- 
tos humanos, y con ello, potenciar el desarrollo de procesos del sujeto que aprende matemáticas, para así, posibilitar el desarrollo de las CM del estudiante.

En lo que atañe a tareas matemáticas, se comparte con Solar (2009), y con Rico y Lupiañez (2008), que las tareas están asociadas al dominio matemático; por tanto, se encuentran articuladas a contenidos, nociones u objetos matemáticos. En el presente caso, la secuencia de tareas propuesta se diseñó teniendo en cuenta el objeto matemático tanto por ciento y el objeto matemático frecuencia absoluta como objeto matemático perteneciente a su estructura conceptual. De otra parte, las tareas tienen un carácter específico, se asocian a expectativas de aprendizaje a corto plazo, las diseña el profesor para promover el desarrollo de procesos asociados a los aspectos del desarrollo humano. Sus objetivos específicos se determinan teniendo presente, entre otros, los diferentes momentos que se producen en la actividad matemática de aprendizaje.

En este caso, tomando como marco de referencia las teorías socioculturales, y con base en la clase de matemáticas como comunidad de aprendizaje en la que se comparten y desarrollan significados matemáticos mediante la comunicación, según Bishop (2005), se diseñó una secuencia de tres tareas, con creciente nivel de complejidad, de acuerdo con los siguientes tres momentos del proceso enseñanza-aprendizaje: acercamiento de los estudiantes a compartir significados matemáticos, compartir significados matemáticos, y desarrollo de significados matemáticos compartidos.

Siguiendo a Solar (2009), el nivel de complejidad de una competencia matemática depende de la complejidad de las tareas y de la complejidad de los procesos matemáticos vinculados con esa competencia. En esta ocasión, se asumieron los niveles de complejidad propuestos por la OCDE (2006), Reproducción, Conexión y Reflexión, para valorar y caracterizar el aspecto cognitivo de las competencias matemáticas. Se tomó distancia conceptual y metodológica de la tendencia a "clasificar" los estudiantes, presente en las pruebas masivas, reconociendo su aporte en lo cognitivo, y se asumieron indicadores de evaluación para valorar y caracterizar los aspectos afectivos y de tendencia de acción.

\subsection{Métodos}

El estudio realizado es una investigación cualitativa con enfoque interpretativo en el que se utilizó la complementariedad de métodos. De la Investigación Acción Participación, IAP, como método de estudio, se adoptaron sus componentes, a saber: i) La investigación como proceso sistemático, controlado, reflexivo y crítico que tiene como objetivo estudiar realidades humanas con una acentuada finalidad práctica; ii) La participación como elemento generador de conocimiento y su práctica como forma de intervención, y iii) El reconocimiento de participantes, en este caso de los estudiantes, como sujetos activos que desarrollan conocimiento y transforman su propia realidad, Eizagirre \& Zabala (s.f., citado por Colmenares, 2012).

El concepto de Participación, central en la IAP, fue esencial en el trabajo de aula especialmente en dos aspectos: movilización de la comunicación de los estudiantes en y con las matemáticas, junto con la resignificación del aprendizaje como discurso con el cual participa en la clase como comunidad de aprendizaje matemático (Sfard, 2008). De acuerdo con lo anterior, se asume la investigación como un proceso dinámico, continuo, complejo y no lineal. A continuación se enlistan las principales actividades realizadas:

a) Revisión de literatura sobre las CM (Ejercicio permanente durante el desarrollo del proceso investigativo).

b) Construcción de un marco teórico y conceptual sobre las CM. 
c) Formulación del problema, la pregunta de investigación, la hipótesis de trabajo y los objetivos.

d) Selección de la muestra. De los 4 cursos en el grado sexto de la Institución educativa Jorge Eliécer Gaitán, se tomó el curso 6A, el cual contaba con 36 estudiantes con edades entre 11 y 13 años.

e) Elaboración del MTP.

f) Diseño y validación de instrumentos para la recolección y sistematización de la información.

g) Concertación con estudiantes de la problemática a utilizar, en este caso el proceso de paz en Colombia, para estudiar el objeto matemático tanto por ciento.

h) Diseño y aplicación de una secuencia de tareas y determinación de las expectativas de aprendizaje, así:

Tarea 1. Opiniones sobre el proceso de paz. Elaboración y aplicación de encuestas a diferentes fuentes humanas: estudiantes, padres de familia, administrativos, profesores y vecinos.

Expectativa de aprendizaje 1. Cuantificar información obtenida de las fuentes humanas mencionadas anteriormente, acerca de las posibilidades de culminar con éxito el proceso de paz que adelantan las FARC y el gobierno nacional en la Habana, Cuba.

Expectativa de aprendizaje 2. Representar la información obtenida para socializarla en clase y, posteriormente, presentarla en la Feria del Emprendimiento que realiza anualmente la Institución Educativa.

Tarea 2. Divulgando el pensar y sentir sobre el proceso de paz.
Expectativa de aprendizaje. Comunicar de manera argumentada, en y con las matemáticas, la información obtenida y sus diferentes formas de representación.

Tarea 3. Corroborando información sobre el proceso de paz.

Expectativa de aprendizaje 1. Proponer transformaciones entre diferentes sistemas semióticos de representación del objeto matemático tanto por ciento, a partir de representaciones incongruentes o incompletas de este.

i) Recolección y sistematización de la información.

j) Análisis y triangulación de la información.

k) Elaboración de conclusiones e informe final.

La información se obtuvo de: documentos producidos por los estudiantes en atención a las demandas planteadas en las tareas, transcripciones de videograbaciones, entrevistas no estructuradas y las notas de la observación participante que consignaron los investigadores en los diarios de campo.

La información sobre la participación de los estudiantes en una secuencia didáctica compuesta por tres situaciones problémicas o tareas de diferente complejidad, se recolectó y se sistematizó en la Tabla de valoración, ver tabla 1. La tabla se elaboró con base en los componentes del MTP mencionado anteriormente. Los indicadores o descriptores establecidos de manera previa, se elaboraron teniendo presente los procesos asociados a las competencias matemáticas razonar y argumentar, representar y comunicar. Se asumieron los aspectos del desarrollo humano: cognitivo, afectivo y tendencia de acción para valorar de manera integral el desarrollo competencial de los estudiantes. De las frecuencias reportadas en los indicadores de la Tabla 1, emergieron los datos que se analizaron y 
que soportan la triangulación que aparece inmediatamente después de la Tabla de valoración.

\section{Resultados y discusión}

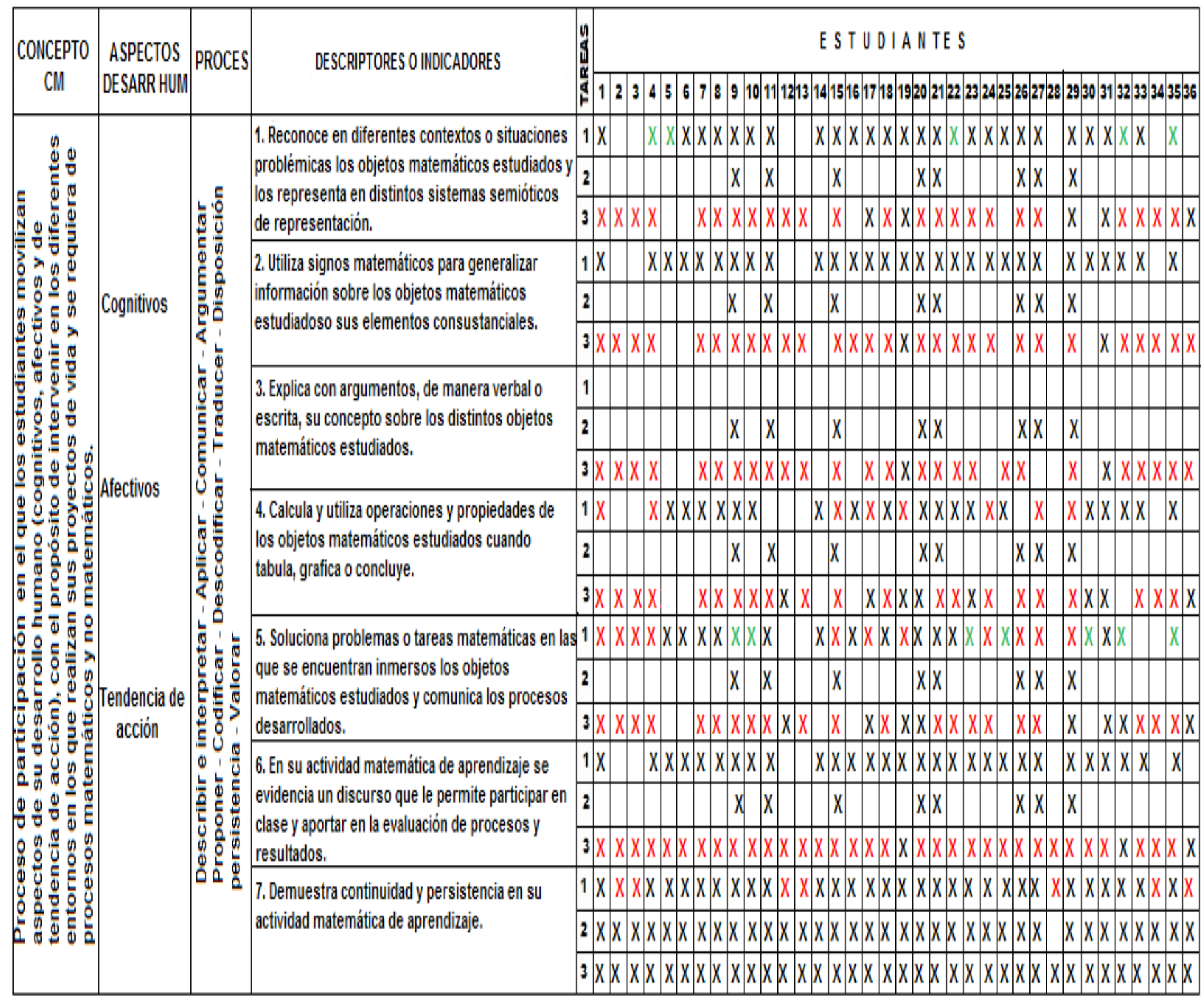

X: Lo cumple X: Lo cumple parcialmente X: No lo cumple

Tabla 1. Tabla de valoración: Sistematización actividad matemática de aprendizaje de los estudiantes.

La triangulación se realizó con base en el seguimiento realizado a la actividad matemática de aprendizaje de los estudiantes, generada por las tareas propuestas. El objetivo de la triangulación fue determinar regularidades y diferencias presen- tadas para describir la actividad matemática de aprendizaje de los estudiantes; en consecuencia, la atención se centró en los indicadores asociados a los procesos consustanciales a las competencias matemáticas razonar y argumentar, representar y 
comunicar. Las tareas mencionadas en la metodología están relacionadas con el aprendizaje de los objetos matemáticos frecuencia absoluta y tanto por ciento. Las tareas contribuyen a desarrollar en el estudiante un discurso matemático centrado en el proceso de compartir y desarrollar el significado matemático, a desarrollar su capacidad comunicativa (preguntar, sustentar, argumentar, proponer) y de participación en la clase como comunidad de aprendizaje.

En correspondencia con las siguientes fases del proceso de enseñanza-aprendizaje: introducción o acercamiento del estudiante a compartir significados de objetos matemáticos, compartir significados de objetos matemáticos y desarrollo de significados compartidos del objeto matemático, las tareas se centraron en: acercar al estudiante al objeto matemático, tarea 1; compartir significados matemáticos, tarea 2; y utilizar los objetos matemáticos en la solución de problemas, tarea 3, para evidenciar que se desarrollaba el significado matemático compartido.

La información obtenida para las tres tareas en el indicador 1 , señala que en la fase de acercamiento a compartir significados del objeto matemático tanto por ciento, tarea 1, 29 de los 36 estudiantes identificaron inicialmente el objeto matemático, frecuencia absoluta, mediante signos no matemáticos; para determinar la cantidad de veces que se presentó una determinada situación, utilizaron palos, equis, cuadros o chulos; luego, los resultados los expresaron en otros sistemas de representación como son: lenguaje simbólico (numeración indo-arábigo), diagrama de barras o una tabla.

La situación cambia en la fase de compartir significados del objeto matemático tanto por ciento, tarea 2. Solo ocho de los 29 estudiantes antes mencionados, a saber: E9, E11, E15, E20, E21, E26, E27 y E29, reconocieron el objeto matemático tanto por ciento en diferentes contextos y lo representaron en diferentes sistemas semióticos de representación: lenguaje verbal oral, lenguaje verbal escrito, lenguaje simbólico, p.ej. 35\%, y como frecuencia relativa con denominador 100, p.ej. 3/100.

En la fase de desarrollo de los significados compartidos del objeto matemático tanto por ciento, tarea 3, o solución de problemas, cinco estudiantes, a saber: E17, E19, E29, E31 y E36, reconocieron el objeto matemático en diferentes contextos y lo representaron en diferentes sistemas semióticos de representación. Llama la atención el caso de E17, E19, E31 y E36, quienes en la fase de compartir significados del objeto matemático tanto por ciento no se manifestaron, no se hizo notoria su participación, pero al momento de utilizar los significados compartidos, lo hicieron de manera correcta; es decir, en su discurso se evidenció aprendizaje y por tanto evolución en procesos consustanciales a las competencias matemáticas representar, comunicar, y razonar y argumentar. El estudiante E29, al atender las demandas solicitadas en cada una de las tres tareas, es el único que reconoció en diferentes contextos o situaciones problémicas los objetos matemáticos frecuencia absoluta y tanto por ciento, y los representó en diferentes sistemas semióticos de representación.

En este mismo indicador 1, de la fase compartir significados, tarea 2, también se encuentran siete estudiantes: E9, E11, E15, E20, E21, E26 y E27, quienes reconocieron en diferentes contextos $O$ situaciones problémicas el objeto matemático frecuencia absoluta y lo representaron en diferentes sistemas semióticos de representación; sin embargo, al momento de desarrollar significados compartidos o de utilizarlo en la solución de problemas, tuvieron dificultades.

La información registrada en el indicador 2 , muestra que el objeto matemático frecuencia absoluta, utilizado para aproximar al estudiante al objeto matemático tanto por ciento, se codificó correctamente por los 29 estudiantes que desarrollaron la tarea 1; es decir, que la cantidad de veces que se presentó una determinada situación, los estudiantes la representaron mediante signos matemáticos como son los números indo-arábigos. 
En concordancia con lo encontrado en el indicador 1, de la fase de compartir significados del objeto matemático tanto por ciento, tarea 2, se encuentra que para el indicador 2 de esta misma tarea, los mismos 8 estudiantes, a saber: E9, E11, E15, E20, E21, E26, E27 y E29, introdujeron en su discurso símbolos matemáticos como 31/100 o $31 \%$, para dar a conocer, comunicar o representar un porcentaje.

Sorprende que en la fase de desarrollo de significados compartidos del objeto matemático tanto por ciento, o aplicación del significado del objeto matemático tanto por ciento en la solución de problemas, tarea 3 del indicador 2, solo dos estudiantes: E19 y E31, expresaron el objeto matemático tanto por ciento mediante el uso de signos matemáticos para informar acerca de él.

En la fase de compartir significados del objeto matemático tanto por ciento, tarea 2 del indicador 3 , en concordancia con lo expresado en los indicadores 1 y 2, participaron 8 estudiantes: E9, E11, E15, E20, E21, E26, E27 y E29, quienes se destacaron por representar mediante signos matemáticos el objeto matemático tanto por ciento, y en este caso, también se destacaron por argumentar o explicar, de manera oral o escrita, lo que entendieron o comprendieron sobre este objeto matemático. Sin embargo, al igual que en el indicador 2, fase desarrollo de significados compartidos, sorprende nuevamente el hecho que ninguno de estos 8 estudiantes argumentó o explicó $y$, mucho menos, utilizó lo comprendido o entendido sobre el objeto matemático para resolver problemas. Situación contraria se presentó con los estudiantes E19 y E31, quienes desarrollaron con éxito la demanda cognitiva de la tarea 3 , a pesar de no hacer parte del grupo de 8 estudiantes referenciados inicialmente.

En lo que atañe al indicador 4: "Calcula y utiliza operaciones y propiedades de los objetos matemáticos estudiados cuando tabula, grafica o concluye", en la fase acercamiento a compartir significados del objeto matemático tanto por ciento, tarea 1, se evidenció que 9 estudiantes: E1, E4, E15, E17, E19, E24, E26, E27 y E29, al determinar la frecuencia absoluta se equivocaron; esto debido a que, aunque aparentemente el proceso de conteo es un proceso sencillo, la falta de concentración durante el conteo y la verificación de lo realizado, según los estudiantes, los condujo al error.

En la fase siguiente de este mismo indicador, compartir significados del objeto matemático tanto por ciento, tarea 2, los estudiantes: E9, E11, E15, E20, E21, E26, E27 y E29, recurrieron a operaciones para realizar cálculos que efectuaron con éxito y les permitió justificar transformaciones intra, y entre diferentes sistemas de representación; lo cual, según Duval (1999), corresponde a tratamientos y conversiones. Por ejemplo, en la encuesta aplicada a 5 administrativos, en la que las frecuencias absolutas fueron 1 y 4 , mediante una división y asumiendo que 5 corresponde al 100\%, E15 encuentra que 1 corresponde al 20\%, y aplicando una multiplicación afirma que 4 corresponde al $80 \%$. Con este tratamiento, el estudiante pasa a elaborar con éxito la correspondiente tabla y diagrama de barras.

Al enfrentarse a la solución de problemas, como expresión del desarrollo de significados compartidos sobre el objeto matemático tanto por ciento, tarea 3 del indicador 4, 1 estudiante de los 8 antes señalados, E20, realiza cálculos para concluir por qué sus compañeros al expresar las frecuencias absolutas en términos de tanto por ciento no lo hicieron correctamente. Lo mismo hacen los estudiantes E12, E17, E19, E23, E31, E32 y E36, quienes en la fase anterior no argumentaron.

Sobre la solución de problemas y de tareas en las que intervienen los objetos matemáticos frecuencia absoluta y tanto por ciento, y la comunicación de procesos desarrollados en tal solución, citados en el indicador 5 , los resultados descritos sobre los indicadores 1 y 4 permiten declarar que en la fase acercamiento a compartir significados de objetos matemáticos, de los 29 estudiantes que participaron en el desarrollo de la tarea 1, 20 soluciona- 
ron adecuadamente la tarea según las demandas planteadas: determinar las frecuencias absolutas en correspondencia con la cantidad de veces que se presenta un evento o situación y representar la información obtenida de maneras diferentes. Los 20 estudiantes realizaron procesos de conteo y representaron mediante números indo-arábigos la información obtenida en al menos dos sistemas diferentes de representación: gráfica, tabular o matemática. Los 9 estudiantes restantes, presentaron dificultades en este proceso aparentemente sencillo de conteo.

En la fase de compartir significados del objeto matemático tanto por ciento, 8 estudiantes: E9, E11, E15, E20, E21, E26, E27 y E29 dieron solución a la tarea 2. Es decir, comunicaron con éxito los procesos matemáticos utilizados para expresar las frecuencias absolutas, obtenidas en la sistematización de la información proveniente de las encuestas aplicadas, en términos de tanto por ciento. Resulta de interés el caso de los estudiantes E15, E26, E27 y E29, quienes en la fase anterior: acercamiento a compartir significados de objetos matemáticos, no solucionaron la tarea; sin embargo, en el proceso de socialización de resultados y presentación de estos de otra manera, tarea 2, reconocieron los errores cometidos, los comprendieron y corrigieron.

En el uso del significado compartido del objeto matemático tanto por ciento, tarea 3, los estudiantes E12, E17, E19, E20, E29, E31, E32 y E36, solucionaron el problema planteado y comunicaron los desarrollos matemáticos implementados para corregir los errores en los que incurrieron sus compañeros, al expresar las frecuencias absolutas en términos de tanto por ciento.

Se destaca el caso de los estudiantes E12 y E36, quienes no realizaron encuesta alguna, por tanto, no participaron en la fase acercamiento a compartir significados; aun así, hacen parte de los 8 estudiantes destacados por el éxito alcanzado en la fase de desarrollo de significados compartidos. Se considera de interés investigativo este hecho y se recomendó su estudio. Caso contrario es lo acontecido con E9, E11 y E21, quienes en las fases anteriores de acercamiento a compartir significado del objeto matemático, atendieron con éxito las demandas planteadas en las tareas correspondientes; sin embargo, en la fase desarrollo de significados compartidos presentaron dificultades que les impidieron expresar las frecuencias absolutas en términos de tanto por ciento. Este hecho, siendo contrario al anterior, también despierta interés y por lo tanto constituye una invitación para profundizar en él.

Notable la participación de E20 en el indicador 5, pues de manera exitosa enfrentó los problemas y atendió las demandas en cada una de las tareas planteadas en las tres fases del proceso didáctico. Fue el único estudiante que en su actividad matemática de aprendizaje solucionó problemas y tareas matemáticas en las que encontró inmersos los objetos matemáticos frecuencia absoluta y tanto por ciento y comunicó los procesos matemáticos desarrollados.

En lo correspondiente a evidencias en el discurso construído para participar en clase y realizar aportes en la evaluación de procesos y resultados, indicador 6 , se encontró en los trabajos realizados en casa, que los 29 estudiantes, en concordancia con lo descrito para los indicadores anteriores, recurrieron a símbolos matemáticos para atender las demandas solicitadas en la tarea 1, fase acercamiento al significado del objeto matemático. En este sentido, en el discurso escrito, como se indicó anteriormente, se halló el uso de los números indo-arábigos para representar el objeto matemático frecuencia absoluta y en el deseo de expresarla de otra manera.

Lo sucedido con el objeto matemático tanto por ciento también se presentó en la fase compartir significados. Los estudiantes E9, E11, E15, E20, E21, E26, E27 y E29, en la socialización o puesta en común sobre la presentación o representación del objeto matemático frecuencia absoluta de otra manera, utilizaron un discurso en el que se eviden- 
ciaron signos matemáticos y notaciones como las antes mencionadas.

En la fase siguiente: desarrollo o uso del significado matemático compartido, la situación es sorprendente. De los 8 estudiantes antes mencionados, ninguno atendió con éxito las demandas planteadas en la tarea 3. Se esperaba que estos estudiantes hicieran uso del discurso, determinaran, argumentaran, explicaran y corrigieran los errores cometidos por algunos de sus compañeros, al expresar las frecuencias absolutas en términos de tanto por ciento. Por el contrario, lo esperado de parte de estos 8 estudiantes se constituyó en un logro para E19, E31 y E36 quienes, para sorpresa de todos, en la fase de compartir significados no participaron. Estos tres estudiantes desarrollaron actividad matemática de aprendizaje en la que identificaron objetos matemáticos (frecuencia absoluta, tanto por ciento), los representaron en varios sistemas de representación semiótica, realizaron cálculos y operaciones para identificar errores y proponer alternativas de solución correctas. Participaron en la clase como comunidad de aprendizaje mediante un discurso que contribuyó en la evaluación de resultados y procesos presentados por sus compañeros.

Para finalizar, de acuerdo con lo acontecido en la secuencia de tareas planteadas y los resultados obtenidos en el indicador 7 , se deduce que los estudiantes responden a las demandas planteadas en las tareas, no renuncian a la actividad matemática de aprendizaje independientemente de los resultados, su participación y nivel de discurso; siempre presentaron sus trabajos y no dejaron de asistir a clases.

Así, 29 estudiantes evidenciaron desarrollo, continuidad y persistencia en su actividad matemática de aprendizaje con el objeto matemático y con los procesos de las competencias matemáticas: Razonar y Argumentar, Representar y Comunicar. Sobresalieron E9, E11, E15, E20, E21, E26, E27 y E29, por su persistencia en la participación; trascendieron la mera asistencia y aprovecharon la interac- ción para dar a conocer de manera argumentada su pensar y proceder, sin que fuera su intención hacerse notar.

\section{Conclusiones}

De manera general, en la actividad matemática de aprendizaje de los estudiantes al enfrentar la secuencia didáctica centrada en el proceso de paz y compuesta por las tareas: opiniones sobre el proceso de paz, tarea 1; divulgando el pensar y sentir sobre el proceso de paz, tarea 2, y corroborando información sobre el proceso de paz, tarea 3, se evidenció el desarrollo de procesos matemáticos y de tendencia de acción, pragmática de uso, para reconocer, representar y usar los objetos matemáticos en la solución de problemas contextualizados. Los estudiantes representan en al menos dos sistemas de representación semiótica, usan los signos matemáticos, calculan, operan y grafican; comunican en y con las matemáticas los procesos desarrollados para solucionar las tareas matemáticas propuestas. Aún así, en su actividad matemática de aprendizaje aún persisten formas de representación semiótica, no propias del lenguaje matemático: palitos, equis, rayas, cuadros, entre otras, que sin duda, limitan un incipiente proceso de matematización y la calidad del discurso matemático. Esto implica deficiencias en la calidad de la participación y en el aporte a la evaluación colectiva de procesos y resultados en la clase como comunidad de aprendizaje.

De otra parte, se hizo notoria la limitada calidad de su discurso matemático, tanto oral como escrito, al valorar procesos y soluciones a las tareas matemáticas que han desarrollado sus compañeros en clase. Es muy posible que los resultados de la tarea 3 puedan también explicarse por la poca familiaridad de los estudiantes con este tipo de actividad matemática y con el nivel de exigencia y responsabilidad que implica una coevaluación. No obstante, ello no influyó para que, durante todo el proceso de intervención, los estudiantes demostraran gusto e inclinación favorable al trabajo individual y en grupo, continuidad y persistencia en 
la construcción y sustentación de sus soluciones, así como a preguntar y buscar orientación en el profesor y en sus compañeros. Esto constituye un clima favorable para consolidar la clase como una comunidad de aprendizaje.

En lo que atañe al MTP de competencia matemática implementado, sus resultados permiten inferir que, trasciende el aspecto cognitivo e incorpora otros aspectos de desarrollo humano (afectivo y de tendencia de acción); requiere del diseño de tareas con crecientes niveles de complejidad determinados por la naturaleza de los objetos matemáticos, los procesos y los contextos en los que estos toman significados. Lo anterior presenta al MTP como un modelo de competencia matemática que contribuye al desarrollo integral del estudiante, ayuda a explicar y comprender las actuaciones del estudiante, la calidad de su participación y de su actividad matemática de aprendizaje.

En consecuencia, el MTP coadyuva a evidenciar dificultades y avances en el desarrollo del pensamiento matemático del estudiante, en su pensar y sentir, y en sus tendencias de acción. De esta manera, el MTP de competencia matemática aquí presentado, puede considerarse como un aporte al reto planteado por Vasco (2013) de reelaborar un concepto fuerte de competencia y estructurar un discurso original, que contribuya de manera significativa al desarrollo de los diferentes aspectos del ser humano.

Además, el MTP contribuye a: i) transformar la organización curricular de las matemáticas escolarizadas al asumir como eje curricular los procesos matemáticos; ii) resignificar la actividad matemática de aprendizaje del estudiante articulándola al desarrollo creciente de su discurso matemático en clase como comunidad de aprendizaje matemático; y iii) reorientar las prácticas de enseñanza del profesor al explicar el proceso de cómo progresan y se movilizan las competencias matemáticas del estudiante y cómo se pueden ir valorando.
El MTP se coloca a disposición del profesorado, los investigadores y las autoridades educativas para que sea criticado y mejorado. Como toda obra humana y con los aportes de la comunidad académica científica nacional e internacional de educadores matemáticos, el MTP continúa en proceso de construcción. Se espera, sea recibido como una contribución que reduzca la sensación en los docentes de no contar con elementos que favorecen el desarrollo de las competencias matemáticas de los estudiantes.

\section{Agradecimientos}

A la vicerrectoría de investigaciones de la Universidad de la Amazonía por haber financiado este proyecto de investigación, según acta 006 del 17 de diciembre de 2012; a los directivos, profesores y estudiantes de la Institución Educativa Jorge Eliécer Gaitán por su rol protagónico durante el proceso investigativo.

\section{Referencias}

Balacheff, N. (2000). Procesos de prueba en los alumnos de matemáticas. Bogotá, Colombia: Una empresa docente y Universidad de Los Andes.

Bishop, A. J. (2005). Una aproximación sociocultural a la educación matemática. Cali, Colombia: Universidad del Valle.

Chacín, B. (2008). Modelo teórico-metodológico para generar conocimiento desde la extensión universitaria. Revista Laurus, 14 (26), 56-88.

Cerón-Álvarez, D. C., Mesa-Laverde, Y. C., \& Rojas-Morales, C. E. (2012). La naturaleza del conocimiento matemático y su impacto en las concepciones del profesor. Revista de Investigación, Desarrollo e Innovación, 2 (2), 49-59. Recuperado de: http://revistas.uptc.edu.co/index.php/investigacion_duitama/article/view/1316 
D'Amore, B., Godino, J., \& Fandiño, M. (2008). Competencias y Matemática. Bogotá, Colombia: Editorial Magisterio.

Colmenares, A. (2012). Investigación-acción participativa: una metodología integradora del conocimiento y la acción. Revista Latinoamericana de Educación. Voces y silencios, 3 (1), 112-115.

Duval, R. (1998). Registros de representación semiótica y funcionamiento cognitivo del pensamiento. Hitt F. (Ed.), Investigaciones en Matemática Educativa II, 173-201. México. Cinvestav.

Duval, R. (1999). Semiosis y pensamiento humano. Registros semióticos y aprendizajes intelectuales. Cali, Colombia: Universidad del Valle.

Espinoza, L., Barbe, Q., Mitrovich, D., Solar, H., Rojas, D., Mateus, C., \& Olguin, P. (2009). Análisis de las competencias matemáticas en primer ciclo: Caracterización de los niveles de complejidad de las tareas matemáticas. (Proyecto FONIDE No: DED0760): Gobierno de Chile. Ministerio de Educación.

Fandiño, M. (2006). Currículo, evaluación y formación docente en matemática. Bogotá, Colombia: Editorial Magisterio.

García-Quiroga, B., Coronado, A., Montealegre-Quintana, L., Giraldo-Ospina, A., Tova-Pizo, B. A., Morales-Parra, S., \& Cortes, D. D. (2012). Competencias matemáticas: un estudio exploratorio en la educación básica y media. Florencia, Colombia: Universidad de la Amazonía.

García-Quiroga, B., Coronado, A., Montealegre-Quintana, L., Giraldo-Ospina, A., Tova-Pizo, B. A., Morales-Parra, S., \& Cortes, D. D. (2013). Competencias matemáticas y actividad matemática de aprendizaje. Florencia, Colombia: Universidad de la Amazonía.

García-Quiroga, B., Coronado, A., \& Giraldo-Ospina, A. (2015). Orientaciones didácticas para el de- sarrollo de competencias matemáticas. Florencia, Colombia: Universidad de la Amazonía.

García-Quiroga, B., \& Coronado, A. (2015). Estudio comparativo de resultados en pruebas saber para estudiantes de $5^{\circ}, 9^{\circ}$ y $11^{\circ}$ del departamento del $\mathrm{Ca}$ quetá y de estudiantes de las instituciones educativas focalizadas en el convenio 021 de 2014. Florencia, Colombia: Patrocinado por UNAD (Documento inédito).

García, G., Acevedo, M., \& Jurado, F. (2003). La dimensión socio-cultural en el criterio de competencia: el caso de matemáticas. Colección cuadernos del seminario en educación No. 5. Bogotá, Colombia: Universidad Nacional de Colombia.

Gascón, J. (2011). ¿Qué problema se plantea el enfoque por competencias? un análisis desde la teoría antropológica de lo didáctico. Recherches en Didactique des Mathématiques, 31 (1), 9-50.

Goldin, G., \& Shteingold, M. (2001). Systems of Representations and the Development of Mathematic al Concepts. En Cuoco y Curcio (Eds.). The Roles of Representation in School Mathematics, 1 -21. Reston, VA: NCTM.

Montes-Miranda, A. J. (2013). La educación básica en Colombia: una mirada a las políticas educativas. Saber, Ciencia y Libertad, 8 (2), 141-155. Recuperado de: http://www.sabercienciaylibertad.com/ojs/ index.php/scyl/article/view/106

Niss, M., \& Højgaard, T. (2011). Competencies and Mathematical Learning ideas and inspiration for the development of mathematics teaching and learning in Denmark: English edition. Recuperado de: hppt://pure.au.dk/portal/files/41669781/THJ11_ MN_KOM_in_english.pdf

OCDE. (2006). PISA 2006. Marco de la evaluación. Conocimientos y habilidades en Ciencias, Matemáticas y Lectura. España: Santillana Educación S.L. 
Piratoba-Gil, R. P., \& Rojas-Morales, C. E. (2014). Cambios en las concepciones iniciales e inducidas sobre la naturaleza de las matemáticas y su didáctica, en estudiantes de un programa de Licenciatura en Matemáticas y Estadística. Revista de Investigación, Desarrollo e Innovación, 5 (1), 32-45. doi: https://doi.org/10.19053/20278306.3140

Kaput, J.J. (1987). Representation Systems and Mathematics. In Janvier (Ed.), Problems ofrepresentation in the teaching and learning of Mathematics, 19-26. Hillsdale: LEA.

Rico, L. (1995). Consideraciones sobre el currículo escolar de Matemáticas. Revista EMA, 1 (1), 4-24.

Rico, R. L., \& Lupiañez, J. (2008). Competencias matemáticas desde una perspectiva curricular. Madrid, España: Alianza.

Riscanevo-Espitia, L. (2016). La teoría de la práctica social del aprendizaje en la formación de profesores de matemáticas. Revista de Investigación, Desarrollo e Innovación, 7 (1), 93-110. doi: https://doi. org/10.19053/20278306.v7.n1.2016.5635

Sierpinska, A. (1995). Understanding in mathematics. Revista Mathematics Education Research Journal, 7 (1), 87-89
Sfard, A. (2008). Aprendizaje de las matemáticas escolares desde un enfoque comunicacional. Cali, Colombia: Universidad del Valle.

Solar, H. (2009). Competencias de modelización y argumentación en interpretación de gráficas funcionales: propuesta de un modelo de competencia aplicado a un estudio de caso. (Tesis doctoral). Universitat Autònoma de Barcelona.

Solar, H. (2011). Propuesta metodológica de trabajo docente para promover competencias matemáticas en el aula, basadas en un Modelo de Competencia Matemática. Institución Adjudicataria: Universidad Católica de la Santísima Concepción. (Proyecto FONIDE N 511091)

Solar, H., García-Quiroga, B., Rojas, F., \& Coronado, A. (2014). Propuesta de un Modelo de Competencia Matemática como articulador entre el currículo, la formación de profesores y el aprendizaje de los estudiantes. Revista Educación Matemática, 20 (2), 33-67.

Stacey, K. (1989). Finding and using patterns in linear generalizing problems. Educational Studies in Mathematics, 20, 147-164.

Vasco-Uribe, C. E. (2013). ¿Disposiciones, capacidades o competencias? XI Congreso pedagógico. Ibagué, Colombia. Recuperado de: http://www. codetol.com/memorias/vasco 